\title{
Case Report: Emergence of Candida auris in the Indian Ocean Region
}

\author{
Yaël Levy, ${ }^{1 \star}$ Guillaume Miltgen, ${ }^{2,3}$ Alexandra Rousseau, ${ }^{4}$ Nathalie Lugagne, ${ }^{4}$ Laura Teysseyre, ${ }^{1}$ Nicolas Traversier, ${ }^{2}$ \\ Marie Desnos-Ollivier, ${ }^{5}$ Nicolas Allou, ${ }^{1,6}$ and Jérôme Allyn ${ }^{1,6}$ \\ ${ }^{1}$ Réanimation Polyvalente, Centre Hospitalier Universitaire La Réunion, Saint-Denis, France; ${ }^{2}$ Laboratoire de Bactériologie, Centre Hospitalier \\ Universitaire La Réunion, Saint-Denis, France; ${ }^{3}$ UMR Processus Infectieux en Milieu Insulaire Tropical (PIMIT), CNRS 9192, INSERM U1187, IRD \\ 249, Université de La Réunion, Saint-Denis, France; ${ }^{4}$ CLIN, Centre Hospitalier Universitaire La Réunion, Saint-Denis, France; ${ }^{5}$ Molecular Mycology \\ Unit, National Reference Center for Invasive Mycoses and Antifungals, Institut Pasteur, CNRS, UMR2000, Paris, France; ${ }^{6}$ Département \\ d'informatique Clinique, Centre Hospitalier Universitaire La Réunion, Saint-Denis Cedex, France
}

\begin{abstract}
Candida auris is an emerging multidrug-resistant yeast that can cause severe infections and spread easily between hospitalized patients, leading to outbreaks in hospital. Here, we report the first four cases of colonization and invasive infection with C. auris reported in the Indian Ocean region. All cases were observed in the French overseas Reunion Island, a very popular destination for European travelers. Three patients had urinary tract or skin colonization, and one had a fatal invasive infection. In three cases, including that of the infected patient, the yeast was not initially identified as $C$. auris, preventing specific hygiene measures to be implemented as suggested in the December 2016 clinical alert to European healthcare facilities. The infected patient likely acquired $C$. auris in the intensive care unit from the first colonized patient. This is the first case of $C$. auris infection and the first potential case of nosocomial transmission of the pathogen to be reported in the French overseas Reunion Island.
\end{abstract}

\section{INTRODUCTION}

The emerging pathogen Candida auris is now considered a threat to global health. ${ }^{1}$ Candida auris is a multidrug-resistant yeast, as most strains are resistant to fluconazole $e^{2,3}$ and have a reduced susceptibility to other triazole antifungals. ${ }^{4}$ Given the high rates of treatment failure and mortality associated with invasive infections, $C$. auris is currently being considered as a highly resistant pathogen. ${ }^{5-9}$ Moreover, the risk of epidemic is high because of $C$. auris's persistence in the environment and resistance to common disinfectants. ${ }^{10}$

In the Indian Ocean region, the prevalence of $C$. auris is unknown because no cases of colonization or infection have been reported to date. Yet given its geographical location, with India to the north and South Africa to the west, the Indian Ocean region is at high risk for the spread of this pathogen. It is also an important tourist destination, notably for Europeans, raising fears that it might help propagate $C$. auris to countries of the north (Figure 1).

Here, we report all cases of $C$. auris observed in Reunion Island to date. We also checked the literature on C. auris in the Indian Ocean region.

\section{METHODS}

French guidelines recommend systematic screening for multidrug-resistant bacterial carriage on hospital admission in patients who have traveled in the past 12 months and on intensive care unit (ICU) admission in all patients whether or not they have spent time abroad. Thus, since 2010, all clinical samples growing yeasts collected in our ICU were routinely screened for $C$. auris. In addition, we retrospectively reviewed all cases of infection by $C$. auris identified in the laboratory and retrospectively reanalyzed all isolates of Candida sp. that have been stored and not identified to the species level before the

*Address correspondence to Yaël Levy, Réanimation Polyvalente, Centre Hospitalier Universitaire La Réunion, Allée des Topazes, 97400, Saint-Denis, La Réunion, France. E-mail: I.yael@hotmail.fr recommendations issued in 2018 to even identify colonizing yeasts. $^{11}$

Candida species were identified using matrix-assisted laser desorption ionization time-of-flight (MALDI-TOF) mass spectrometry and MALDI Biotyper IVD ${ }^{\circledR}$ database. All isolates identified as $C$. auris and responsible for colonization or infection were then stored and sent to the French National Reference Center for Invasive Mycoses and Antifungals (NRCMA, Institute Pasteur, Paris, France), for confirmation of identification. The minimal inhibitory concentrations (MICs) were determined by the European Committee on Antimicrobial Susceptibility Testing method. ${ }^{12}$ Short tandem repeat (STR) typing assay was used to determined geographic clade of the isolates. ${ }^{13}$

\section{CASES (TABLE 1)}

Four patients were colonized or infected with $C$. auris between 2010 and 2019. All patients were hospitalized in the same ICU. Informed consent to publish was obtained from cases 1,3 , and 4 and from the family of the dead case 2 .

Case 1. A 76-year-old woman, who had spent 6 weeks in Mauritius for holidays, was admitted to the ICU in 2015. Her past medical history included untreated chronic lymphocytic leukemia and chronic heart failure. In Mauritius, she was hospitalized in the ICU for 1 month for dyspnea. A broadspectrum antibiotic regimen of meropenem and levofloxacin was initiated. Her clinical condition deteriorated, prompting her medical transfer to the ICU of Reunion Island where she was diagnosed with Legionella pneumophila. New infectious complications required urine culture, which grew Candida species later (in 2019), identified as $C$. auris but not stored. The patient was considered to be colonized in the urinary tract, and no antifungal susceptibility testing was performed, and no antifungal drug was prescribed. The patient was discharged from the ICU on day 49.

Case 2. In April 2015, a 36-year-old man with paroxysmal nocturnal hemoglobinuria treated with eculizumab was admitted to the ICU for coma due to cerebral thrombophlebitis with intracranial hypertension. No travel history was reported. The patient was diagnosed with $C$. auris fungemia (positive 


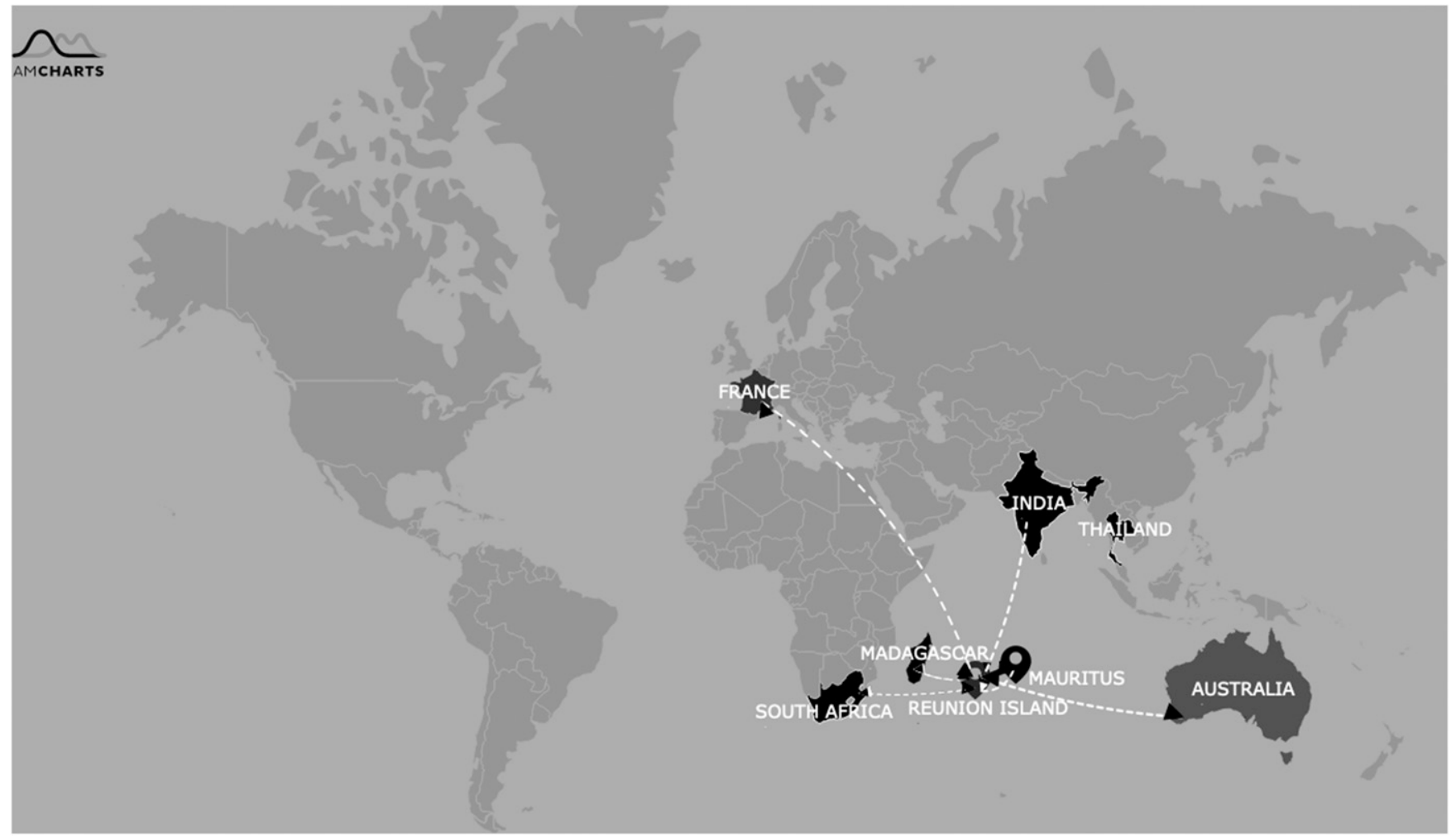

FIGURE 1. Indian Ocean region: Reunion Island is directly connected to European continent via several daily flights to Paris. White dotted line showed Reunion Island's daily flights.

blood and central venous catheter cultures). Caspofungin was prescribed for a period of 13 days (loading dose of $70 \mathrm{mg}$, followed by $50 \mathrm{mg} /$ day). The persistence of positive blood cultures prompted the addition of liposomal amphotericin B ( $350 \mathrm{mg} /$ day), after 5 days of caspofungin monotherapy. The diagnosis of C. auris endocarditis was made based on the discovery of vegetation of the mitral valve by transoesophageal echocardiography and the persistence of positive blood cultures until the patient died on day 19 from refractory intracranial hypertension. The species was confirmed at the National reference center for invasive mycoses and antifungals (NRCMA) (\#Centre National de Référence des Mycoses Invasives et Antifongiques [CNRMA] 15.337). The isolate exhibiting high fluconazole $\mathrm{MIC}$ (> $64 \mathrm{mg} / \mathrm{L})$ was found to belong to the Indian clade $\mathrm{I}$.

Case 3. In 2016, a 30-year-old man with no recent travel and no past medical history apart from chronic alcohol abuse was admitted to the ICU for pneumonia due to L. pneumophila. Hospitalization was complicated by multiple nosocomial infections, which required successive treatments. On day 30 , Candida sp., later (in 2019) identified as C. auris, was recovered from the patient's skin but neglected. The patient was discharged from the ICU on day 54 and from hospital on day 100.

Case 4. In 2019, an 83-year-old man who had traveled in Saudi Arabia and India for 1 month was admitted to an Indian ICU for septic shock due to pneumonia. The patient, who had no past medical history, was treated with a broad-spectrum antibiotic regimen of penicillin, meropenem, and minocycline. He was discharged from the Indian ICU after 2 weeks. He returned home to Reunion Island, and 3 weeks later, he was admitted to the ICU for necrotizing pleuropneumonia of the right lobe with septic shock. Bronchoalveolar lavage fluid and blood cultures revealed the presence of carbapenemresistant Klebsiella pneumoniae. Vancomycin-resistant Enterococcus faecium was detected via rectal swab. Urine culture grew yeast identified as $C$. auris with no other positive body sites. The patient's condition improved rapidly under antibiotics therapy, but no antifungal drug was prescribed. He was discharged from the ICU on day 2 and from hospital on day 10 . The $C$. auris isolate was sent to the NRCMA (\#CNRMA19.95) where the species was confirmed. The isolate also belonged to Indian clade I, and antifungal susceptibility testing showed high fluconazole and flucytosine MICs (MICs > $64 \mathrm{mg} / \mathrm{L}$ ).

\section{LITERATURE REVIEW AND DISCUSSION}

These cases suggest that $C$. auris has been present in the Indian Ocean region for at least 5 years. As in other reports, C. auris was here found mainly in immunocompromised ICU patients as a skin or urinary tract colonization. ${ }^{4,14}$

In the literature, most cases reported of $C$. auris colonization and infection concern the Indian subcontinent. ${ }^{4,14}$ Patients hospitalized in Indian hospitals are at very high risk of $C$. auris colonization or infection because $C$. auris was recovered in the majority of ICUs (19/27) studied in India where it represents $5.2 \%$ of all Candida spp. isolates. ${ }^{15}$ Most cases of C. auris invasive infection occur in critically ill patients with major risk factors ${ }^{14}$ and are associated with a mortality rate above $50 \%{ }^{1}$

Before our study, no case of $C$. auris had been documented in the Indian Ocean region. The four cases reported here were all observed in Reunion Island. Two of the cases occurred after a stay in India (Case 4) and Mauritius (Case 1). Case 3 remains 
TABLE 1

Characteristics of cases and risk factors for Candida auris invasive infection

\begin{tabular}{|c|c|c|c|c|}
\hline Case & 1 & 2 & 3 & 4 \\
\hline Year/month & 2015/April & 2015/April & 2016/May & 2019/June \\
\hline Gender, age (years) & Female, 76 & Male, 36 & Male, 30 & Male, 83 \\
\hline Comorbidity & $\begin{array}{l}\text { Chronic lymphocytic leukemia } \\
\text { and chronic heart failure }\end{array}$ & Hypertension & None & None \\
\hline $\begin{array}{l}\text { Country visited in the previous } \\
\text { year }\end{array}$ & Mauritius & None & None & $\begin{array}{l}\text { India and Saudi } \\
\text { Arabia }\end{array}$ \\
\hline $\begin{array}{l}\text { Recent intake of broad-spectrum } \\
\text { antibiotics }\end{array}$ & Yes & No & Yes & Yes \\
\hline Total parenteral nutrition & No & No & No & No \\
\hline Urinary catheterization & Yes & Yes & Yes & Yes \\
\hline Postoperative drain placement & No & No & No & No \\
\hline Vascular surgery & No & No & No & No \\
\hline $\begin{array}{l}\text { Type of disease or location of } \\
\text { isolate }\end{array}$ & Colonization (urinary tract) & $\begin{array}{l}\text { Candidemia and central venous } \\
\text { catheter tip }\end{array}$ & $\begin{array}{l}\text { Colonization } \\
\text { (skin) }\end{array}$ & $\begin{array}{l}\text { Colonization (urinary } \\
\text { tract) }\end{array}$ \\
\hline Other Candida species & C. tropicalis & No & $\begin{array}{l}\text { Candida } \\
\text { albicans }\end{array}$ & $\begin{array}{l}\text { Candida parapsilosis } \\
\text { C. tropicalis }\end{array}$ \\
\hline Antifungal resistance & Not available & $\begin{array}{l}\text { Fluconazole } \\
\text { 5-Flucytosine }\end{array}$ & Not available & $\begin{array}{l}\text { Fluconazole } \\
\text { 5-Flucytosine } \\
\text { Voriconazole }\end{array}$ \\
\hline Treatment & 0 & Echinocandin 13 days & 0 & 0 \\
\hline Death & No & Yes & No & No \\
\hline
\end{tabular}

C. tropicalis $=$ Candida tropicalis.

unexplained in terms of the origin of contamination, except for a stay in an ICU. However, no other patient staying at the same time was retrospectively identified as a carrier of the yeast or having recently traveled to a country at risk for $C$. auris contamination.

As often reported, both isolates tested exhibited high fluconazole and flucytosine MICs in line with recent studies published. ${ }^{16,17}$ Susceptibility to echinocandins and amphotericin B was considered normal (Table 2). Interestingly, the three 5 -flucytosine-resistant $C$. auris isolates $(6 \%)$ analyzed by Lockhart et al. all came from India, which is also the case for the French isolates including ours (for Case 4), both belonging to the Indian clade. ${ }^{16,18}$ Moreover, both isolates (Case 2 and Case 4) have the same genotype (STR genotype 17) which is the main genotype observed in the South Asian clade I (Table 3). ${ }^{13}$

A first clinical alert on the emergence and spread of $C$. auris was issued in 2016 by the CDC. ${ }^{19}$ In March 2018, the French network of Hospital Infection Control Committees also published a clinical alert, ${ }^{11}$ prompting the implementation of specific hygiene measures for the prevention of $C$. auris transmission. After national alert, ${ }^{11}$ all patients admitted in our ICU were screened (i.e., rectal swab) for C. auris and for multidrug-resistant pathogens. All screen-positive patients were isolated, and specific measures of hygiene were set up. The following measures were recommended: all patients colonized or infected with $C$. auris must be isolated in a single room; standard precautions including hand hygiene must strictly adhered to (handwash with soap and water to remove visible soiling followed by alcohol hand rub during care activities, and alcohol hand rub in the absence of visible soiling between care activities); and the bed space and/or room of colonized or infected patients must be terminally cleaned after discharge using a hypochlorite at $1,000 \mathrm{ppm}$ of available chlorine (i.e., a concentration higher than that routinely used). Non-environmental screening of healthcare workers was carried out, but all close contacts (patients in the same ICU) were screened and isolated until three consecutive negative screens were obtained (at least 24 hours apart). Before these recommendations, the ICU was disinfected with a product composed of quaternary ammonium compounds unable to eradicate $C$. auris. ${ }^{20,21}$ We hypothesized that Case 2 was contaminated via the environment on the grounds that he was diagnosed before the implementation of specific hygiene measures and was hospitalized in the same ICU room as Case 1, but after Case 1 left (4 days later). The strain from Case 1 being unavailable for analysis, we tested this hypothesis by retrospectively comparing five spectra obtained from the two patients suspected of cross-transmission with MALDI-TOF mass spectroscopy (Bruker Daltonics, Bremen, Germany). Two spectra from Case 1 were added to the MALDI Biotyper IVD database (Bruker Daltonics) to create a new main spectrum profile. Three spectra from Case 2 were then compared with the new database. In each instance, the two spectra from Case 1 matched the first two positions of the top 10 ranking with a logscore value $>2.0$ (Figure 2). Although the discriminating power of MALDI-TOF to finely type bacteria or yeasts is limited, it is now recognized that the mass spectrometry is also able of strain typing and notably to classify with a good reliability the

TABLE 2

Antifungal resistance of Candida auris isolates (Cases 2 and 4)

\begin{tabular}{|c|c|c|c|c|c|c|c|}
\hline \multirow[b]{2}{*}{ Isolate } & \multicolumn{7}{|c|}{ Antifungal (minimal inhibitory concentration mg/L) } \\
\hline & Fluconazole & Voriconazole & Posaconazole & Amphotericin B & 5-Flucytosine & Caspofungin & Micafungin \\
\hline 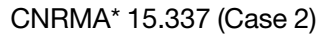 & $\geq 64$ & 0.125 & $\leq 0.014$ & 0.25 & $\geq 64$ & 0.015 & 0.03 \\
\hline 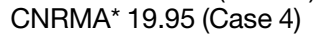 & $\geq 64$ & 1 & 0.06 & 0.125 & $\geq 64$ & 0.03 & 0.03 \\
\hline
\end{tabular}

${ }^{*}$ CNRMA: French National Reference Center for Invasive Mycoses and Antifungals (Pasteur Institute, Paris). Boldface indicates a significant result. 
TABLE 3

Short tandem repeat genotype profile for isolates of Cases 2 and 4

\begin{tabular}{lccccccccccccccc}
\hline & & \multicolumn{1}{c}{ Locus } \\
\cline { 2 - 10 } Isolate & STR genotype & M2a & M2b & M2c & M3-la & M3-lb & M3-Ic & M3-Ila & M3-Ilb & M3-Ilc & M9a & M9b & M9c \\
\hline Case 2 & 17 & 66 & 19 & 9 & 30 & 10 & 18 & 36 & 29 & 22 & 18 & 11 & 9 \\
Case 4 & 17 & 66 & 19 & 9 & 30 & 10 & 18 & 36 & 29 & 22 & 18 & 11 & 9 \\
\hline
\end{tabular}

microorganisms in clonal complexes such as ST (sequence type) for bacteria. ${ }^{22-24}$ This typing method is based on specific protein peaks. Thus, the strains of the Cases 1 and 2 are closer together than the other $C$. auris spectra in the Bruker mass spectrometer database. This finding supported the hypothesis of nosocomial transmission from Case 1 to Case 2. Accordingly, Case 2 was retrospectively reported to the health authorities, as the first potential case of nosocomial transmission documented in France. It should be noted, however, that this transmission cannot be confirmed by a molecular typing method because the $C$. auris strain isolated from Case 1 in 2015 is no longer available.
Unsurprisingly, C. auris has been present in the Indian Ocean area for several years. Thanks to its central location in Indian Ocean and thanks to its technical platform, Reunion Island supports the surveillance and control actions of $C$. auris that were already implemented on the island as a result of these cases.

Received June 28, 2020. Accepted for publication September 27, 2020.

Published online December 14, 2020.

Acknowledgment: We thank Arianne Dorval and Florence Marche for their assistance during the preparation of the manuscript.

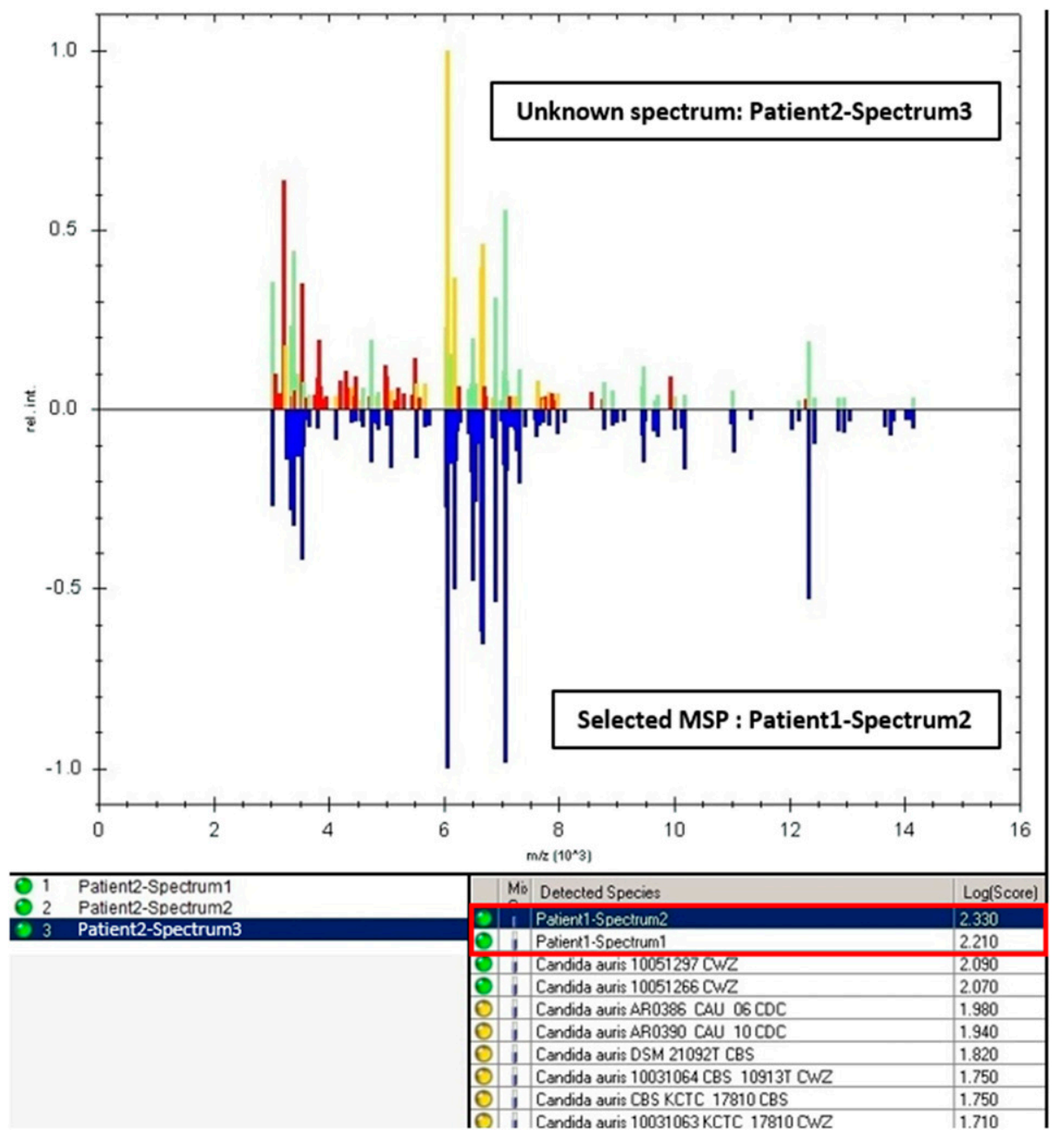

FIGURE 2. Comparison of a spectrum from Case 2 (Spectrum 3) to the modified Bruker database (MALDI Biotyper IVD database version 2.37712. Main spectrum profile modified with the addition of two spectra from Case 1 for a total of 11 Candida auris spectra). The spectra of Case 1 matched the first two positions (log-score values: 2.33 and 2.210) of the top 10 ranking. Our tested spectrum (or unknown spectrum) is represented in the upper part of each panel, and the reference spectrum selected from the database appears in blue in the lower part of each panel (thresholds of composite correlation index: $<0.80$, red: low matching; $0.80-0.90$, yellow: moderate matching; $>0.90$, green: high matching). This figure appears in color at www.ajtmh.org. 
Authors' addresses: Yaël Levy, Laura Teysseyre, Nicolas Allou, and Jérôme Allyn, Intensive care unit, Reunion University Hospital Center, Saint-Denis, France, E-mails: I.yael@hotmail.fr, laura.teysseyre@ gmail.com, nicolas.allou@hotmail.fr, and jerome.allyn@chu-reunion.fr. Guillaume Miltgen and Nicolas Traversier, Department of bacteriology, Reunion University Hospital Center, Saint-Denis, France, E-mails: guillaume.miltgen@chu-reunion.fr and nicolas.traversier@chu-reunion.fr. Alexandra Rousseau and Nathalie Lugagne, Nosocomial infection unit, Reunion University Hospital Center, Saint-Denis, France, E-mails: alexandra.rousseau@chu-reunion.fr and nathalie.lugagne@chureunion.fr. Marie Desnos-Ollivier, Department of Mycology, Pasteur Institute, Paris, France, E-mail: marie.desnos-ollivier@pasteur.fr.

\section{REFERENCES}

1. Jeffery-Smith A, Taori SK, Schelenz S, Jeffery K, Johnson EM, Borman A; Candida auris incident Management Team, Manuel R, Brown CS, 2018. Candida auris: a review of the literature. Clin Microbiol Rev 31: e00029-17.

2. Schelenz $S$ et al., 2016. First hospital outbreak of the globally emerging Candida auris in a European hospital. Antimicrob Resist Infect Control 5: 35.

3. Magobo RE, Corcoran C, Seetharam S, Govender NP, 2014. Candida auris-associated candidemia, South Africa. Emerg Infect Dis 20: 1250-1251.

4. Chowdhary A et al., 2014. Multidrug-resistant endemic clonal strain of Candida auris in India. Eur J Clin Microbiol Infect Dis 33: 919-926.

5. CDC, 2019. General Information about Candida auris | Candida auris | Fungal Diseases. Available at: https://www.cdc.gov/ fungal/candida-auris/candida-auris-qanda.html. Accessed November 19, 2019.

6. European Centre for Disease Prevention and Control, 2018. Rapid Risk Assessment: Candida auris in Healthcare Settings - Europe. Available at: https://www.ecdc.europa.eu/en/publications-data/ rapid-risk-assessment-candida-auris-healthcare-settingseurope. Accessed November 19, 2019.

7. GOV.UK, 2016. Candida auris: Laboratory Investigation, Management and Infection Prevention and Control. Available at: https://www.gov.uk/government/publications/candida-aurislaboratory-investigation-management-and-infection-preventionand-control. Accessed November 19, 2019.

8. Snyder GM, Wright SB, 2019. The epidemiology and prevention of Candida auris. Curr Infect Dis Rep 21: 19.

9. Bidaud AL, Chowdhary A, Dannaoui E, 2018. Candida auris: an emerging drug resistant yeast-a mini-review. J Mycol Med 28: 568-573.

10. Ledwoch K, Maillard JY, 2018. Candida auris dry surface biofilm (DSB) for disinfectant efficacy testing. Materials (Basel) 12: 18.

11. Maurand A, Gagnaire J, Berthelot P, 2018. Candida auris: émergent, multi-résistant, nosocomial, transmissible. Bulletin National du Centre de Prévention des infections associées aux soins (CPias) $n^{\circ} 10$. Available at: http://www.cpias.fr/bulletin/ 2018/10/10-pdf/10-tous_les_articles.pdf.
12. Arendrup MC, Cuenca-Estrella M, Lass-Flörl C, Hope W, 2012. EUCAST technical note on the EUCAST definitive document EDef 7.2: method for the determination of broth dilution minimum inhibitory concentrations of antifungal agents for yeasts EDef 7.2 (EUCAST-AFST)*. Clin Microbiol Infect 18: E246-E247.

13. de Groot T, Puts Y, Berrio I, Chowdhary A, Meis JF, 2020. Development of Candida auris Short tandem repeat typing and its application to a global collection of isolates. mBio 11: e0297119.

14. Rudramurthy SM et al., 2017. Candida auris candidaemia in Indian ICUs: analysis of risk factors. J Antimicrob Chemother 72: 1794-1801.

15. Chakrabarti A et al., 2015. Incidence, characteristics and outcome of ICU-acquired candidemia in India. Intensive Care Med 41: 285-295.

16. Lockhart SR et al., 2017. Simultaneous emergence of multidrugresistant Candida auris on 3 continents confirmed by wholegenome sequencing and epidemiological analyses. Clin Infect Dis 64: $134-140$.

17. Arendrup MC, Prakash A, Meletiadis J, Sharma C, Chowdhary A, 2017. Comparison of EUCAST and CLSI reference microdilution MICs of eight antifungal compounds for Candida auris and associated tentative epidemiological cutoff values. Antimicrob Agents Chemother 61: e00485-17.

18. de Groot T, Puts Y, Berrio I, Chowdhary A, Meis JF, 2019. Development of Candida auris microsatellite typing and its application on a global collection of isolates. bioRxiv. 792663. doi: 10.1101/792663.

19. Vallabhaneni $S$ et al., 2016. Investigation of the first seven reported cases of Candida auris, a globally emerging invasive, multidrug-resistant fungus-United States, May 2013-August 2016. MMWR Morb Mortal Wkly Rep 65: 1234-1237.

20. Moore G, Schelenz S, Borman AM, Johnson EM, Brown CS, 2017. Yeasticidal activity of chemical disinfectants and antiseptics against Candida auris. J Hosp Infect 97: 371-375.

21. Welsh RM, Bentz ML, Shams A, Houston H, Lyons A, Rose LJ, Litvintseva AP, 2017. Survival, persistence, and isolation of the emerging multidrug-resistant pathogenic yeast Candida auris on a plastic health care surface. J Clin Microbiol 55: 2996-3005.

22. Sauget M, Valot B, Bertrand X, Hocquet D, 2017. Can MALDI-TOF mass spectrometry reasonably type bacteria? Trends Microbiol 25: 447-455.

23. Quéro L, Courault $P$, Cellière B, Lorber $S$, Jany JL, Puel O, Girard V, Vasseur V, Nodet P, Mounier J, 2020. Application of MALDITOF MS to species complex differentiation and strain typing of food related fungi: case studies with Aspergillus section flavi species and Penicillium roqueforti isolates. Food Microbiol 86: 103311.

24. Al-Hatmi AMS, Normand AC, van Diepeningen AD, Hendrickx $M$, de Hoog GS, Piarroux R, 2015. Rapid identification of clinical members of Fusarium fujikuroi complex using MALDI-TOF MS. Future Microbiol 10: 1939-1952. 